\title{
The in situ structure of the $\mathrm{L3}$ and $\mathrm{L4}$ proteins of the large subunit of E. coli ribosomes as determined by nuclear spin contrast variation
}

\author{
J. ZHAO and H.B. STUHRMANN
}

Abt. WS, GKSS-Research Center, 2054 Geesthacht, Germany

\begin{abstract}
Polarized Neutron scattering from dynamically polarized targets has been used to determine the in situ structure of the proteins L3 and L4 of the large subunit of E.coli ribosome. Both proteins $\left(M \approx 2.3 \times 10^{4}\right)$ have an elongated shape and radii of gyration of about $19 \AA$. The orientation of the proteins with respect to that of the ribosomal subunit has been determined. The distance between the centers of mass of L3 and L4 amounts to $125 \pm 10 \AA$.
\end{abstract}

\section{INTRODUCTION}

Hydrogen is the most abundant element in biological matter. Unlike many other elements it has a negative neutron scattering amplitude $\left(-0.374^{*} 10^{-12} \mathrm{~cm}\right.$ ). Its heavier isotope ${ }^{2} \mathrm{H}$ (or $\mathrm{D}=$ deuterium) has a scattering length of $+0.66^{*} 10^{-12} \mathrm{~cm}$ which is similar to those of other elements in biological matter. Thus, isotopic exchange of $\mathrm{H}\left(=^{1} \mathrm{H}\right)$ by $\mathrm{D}$ has a strong influence on the intensity of neutron scattering from hydrogenous material. An even more pronounced change of the scattering length is when polarized neutrons are scattered by polarized protons. Using completely polarized neutron beam, the amplitude of coherent scattering from totally polarized protons varies from $-1.83^{*} 10^{-12} \mathrm{~cm}$ to $+1.082^{*} 10^{-12} \mathrm{~cm}$ when the direction of neutron and proton spins varies from antiparallel to parallel. The nuclear polarization has much smaller effect on deuteron scattering amplitude. We expect therefore a strong change of the scattering density of protonated material in polarized neutron scattering experiments and to a much smaller extent from deuterated matter. Proton clusters can be well contrasted with a deuterated matrix and even more if their nuclear spins are polarized. This is the basis of nuclear spin contrast variation.

\section{The Instrument}

The neutron small-angle scattering instrument SANS-1 of GKSS Research Center is shown in Fig.1. Many of its components are similar to other small-angle instruments. We just mention a few features here. The neutrons are obtained from a $5 \mathrm{MW}$ swimming pool reactor with a cold source. The cold neutron flux before the velocity selector is $2 \times 10^{8} \mathrm{n} / \mathrm{sec} / \mathrm{cm}^{2}$. The relative width of wavelength spectrum after the selector is $10 \%$. The collimation length can be changed to $2,3,7$ and 9 meters. The sample-detector distance can be changed continuously from 0.7 to 10 meters

In addition, the neutron beam is polarized by total reflection from magnetized surfaces ${ }^{[1]}$. The polarization of the neutrons is maintained by a magnetic guide field from a sequence of solenoids in the collimator tube. A flat coil spin flipper half way between the neutron polarizer and the sample is used to invert the neutron polarization. 


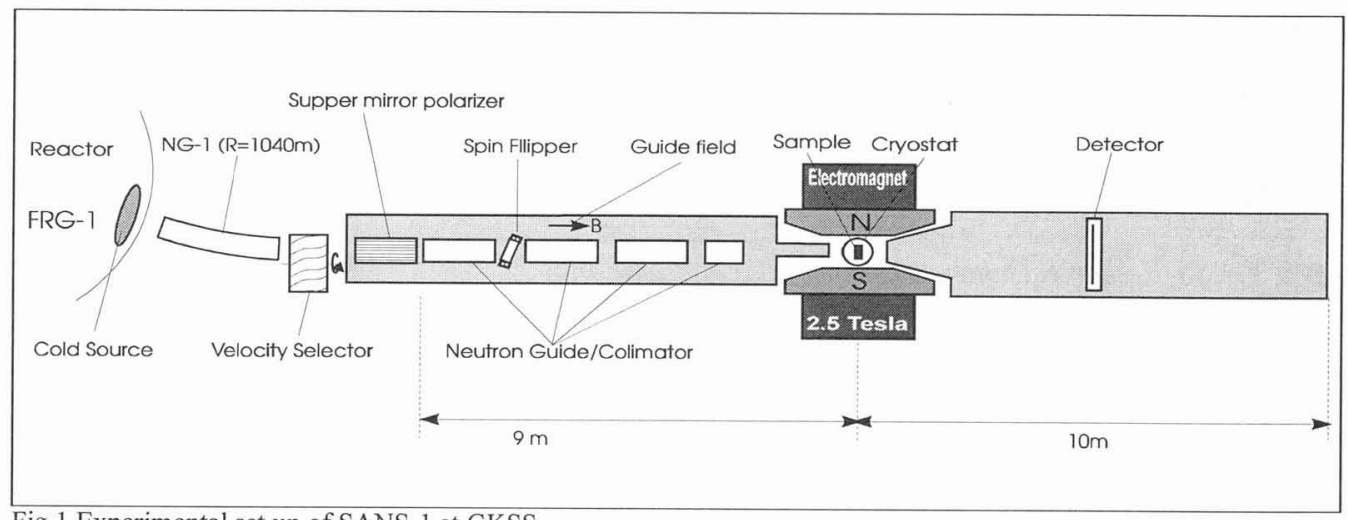

Fig.1 Experimental set up of SANS-1 at GKSS

The polarization of the nuclear spins is achieved by dynamic nuclear spin polarization (DNP). In a strong magnetic field $(2.5 \mathrm{~T})$ and at temperatures below $1 \mathrm{~K}$, the nuclear spins are polarized by irradiation of 4 $\mathrm{mm}$ microwaves in the presence of paramagnetic impurities. As paramagnetic impurities we take an organic compound of $\mathrm{Cr}(\mathrm{EHBA}-\mathrm{Cr}(\mathrm{V}))$ which has a free electron spin of $1 / 2$. By choosing microwave frequencies slightly below or above the peak of the $\mathrm{Cr}(\mathrm{V}) \mathrm{EPR}$, nuclear spins can be driven to align in the positive and the negative direction respectively. All nuclei with non-zero spin will be polarized in this way to a different extent depending on their nuclear magnetic moment. In practice proton spin polarizations of 70 to $95 \%$ are reached after some hours of microwave irradiation. The deuterons then will be polarized to about 20 to $30 \%$ and the polarization of nitrogen nuclei is expected to reach about half this value. Fig 2 shows the essential components of DNP.

The nuclear polarization is observed by continuos wave NMR. The NMR peaks of protons and deuterons are 106.3 and 16.3 $\mathrm{MHz}$ respectively in the $2.5 \mathrm{~T}$ field used. The same resonance circuit is also used for selective nuclear depolarization. By a high power rf sweep across the proton NMR-signal, the proton spins will then be depolarized, whereas the other nuclear spins are not affected. A deuterated sample then would become a deuteron target. Similarly, a proton target can be produced by selective depolarization of the deuteron spins of a dynamic polarized target.

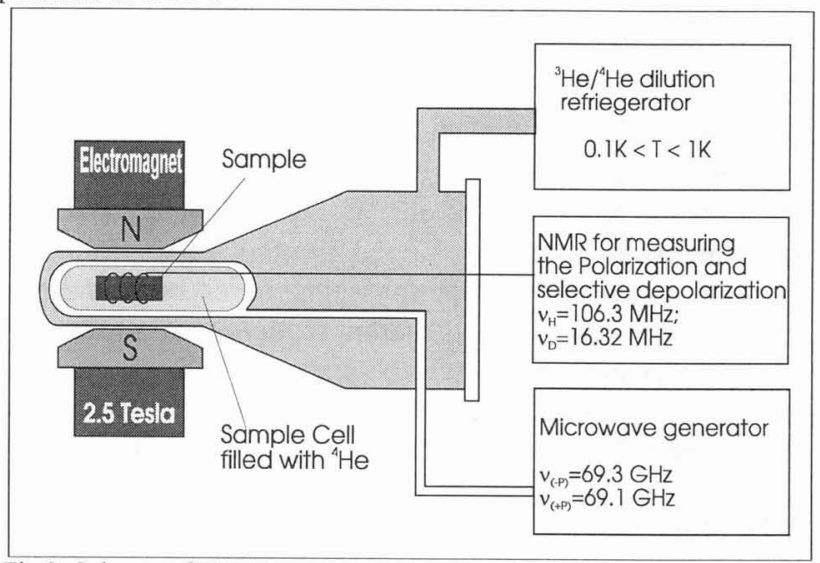

Fig.2 Schema of DNP set up.

\section{The Ribosome}

Ribosomes are the protein factories in each living cell. The genetic information is transcribed from DNA to messenger-RNA (mRNA), and then 'read' by the ribosome to produce proteins. The ribosome consists of two subunits, the small (30S) and the large (50S) subunit. This paper deals with the large subunit which consist of 2 rRNAs and 34 ribosomal proteins named as L1 to L34. Our interest here concerns L3 and L4. Both have a molecular weight of about $22 \mathrm{kda}$, which is two orders of magnitude smaller than that of the total partical $(\mathrm{MW} \approx 1400 \mathrm{kda})$. The deuterated $50 \mathrm{~S}$ subunits with protonated ribosomal proteins $\mathrm{L} 3$ and/or L4 are prepared by total reconstitution ${ }^{[2],[3]}$. 


\section{Measurements}

Five different samples were measured by polarized neutron scattering from polarized target:

(1). Deuterated 50S subunit

(2). dito but with protonated L3

(3). dito but with protonated L4

(4). dito but with protonated $\mathrm{L} 3+\mathrm{L} 4$

(5). Deuterated solvent (glycerold $_{8}: \mathrm{D} 2 \mathrm{O}=11: 9$ )

The of the 50S in the sample is around $1 \mathrm{wt} . \%$. The EHBACr(V) complex added to the sample is about 0.8 wt.\%. Each sample is measured at following different conditions: (1) unpolarized, (2) only protons polarized, (3). only deuterons polarized and (4). both protons and deuterons polarized. The typical measuring time for one sample is about 1 week.

\section{Data Treatment}

Generally the spin dependent small angle neutron scattering can be expressed as following ${ }^{[4]}$ :

$$
I(Q)=|U(Q)|^{2}+2 P_{n} \operatorname{Re}\left[U(Q) V^{*}(Q)\right]+|V(Q)|^{2}
$$

where $P_{n}$ is the neutron spin polarization. The nuclear spin polarization $P_{1}$ is included in $V(Q)$ whereas $U(Q)$ does not depend on spin polarization. Hence $\operatorname{Re}\left[\mathrm{U}(\mathrm{Q}) \mathrm{V}^{*}(\mathrm{Q})\right]$ varies with $\mathrm{P}_{\mathrm{I}}$ and $|\mathrm{V}(\mathrm{Q})|^{2}$ varies with $P_{I}^{2}$.

The three basic scattering functions in eq. (1) are obtained in the following way: $|\mathrm{U}|^{2}$ is the scattering of the unpolarized target. From two measurements with opposite neutron spin polarizations, [UV] is obtained as the difference between these two measurements and $|\mathrm{V}|^{2}$ is the sum of them subtracting $|\mathrm{U}|^{2}$. As an example, the basic scattering functions of sample No.2, is shown in Fig 3.

The curves reflect the amplitude of the deuterated ribosomal subunit which has a very low contrast with respect to the deuterated solvent and the amplitude of the protonated L3 protein which has a very high contrast both in $U(Q)$ and in $V(Q)$. The following analysis aims at the determination of the in situ structure of the L3 protein, i.e. of its shape and its position inside the 50S subunit. For this purpose we introduce the low resolution model of the $50 \mathrm{~S}$ subunit as determined by electron microscopy ${ }^{[5]}$. It is considered as known. The unknown parameters are the site and the shape of the L3 protein. In a first step

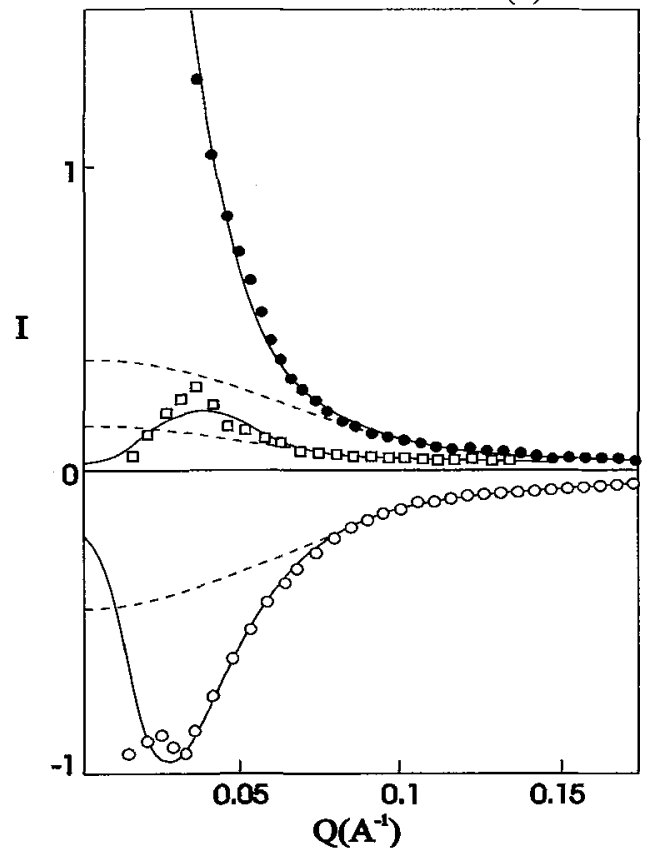

Fig 3. Basic scattering functions of $\mathrm{L} 3+\mathrm{L} 4$ :

$$
\rightarrow|\mathrm{U}|^{2} ; 0 \rightarrow 2 \mathrm{Re}|\mathrm{UV}| ; \bullet \rightarrow|\mathrm{V}|^{2}
$$

The solid lines are fitted curves and the dotted lines are calculated intensity of the labels alone. we determine the site of the L 3 protein. The L3

protein is described as a spherical particle with the radius $R$. The coordinates of its center of mass are varied all over the volume of the $50 \mathrm{~S}$ subunit and the corresponding calculated basic scattering functions are compared with those obtained from the experiment. The expression to be minimzed is

$$
\delta=\sum\left|I(Q)_{\text {measured }}-I(Q)_{\text {calculated }}\right|
$$


Fig 4. shows the contours of $\mathrm{L} 3$ where $\mathrm{L} 3$ is taken as a sphere. There is only one region where $\delta$ reaches a minimum, i.e. the determination of the site of $\mathrm{L} 3$ is unique.

In a further step we allow for an elongated shape of L3 which is described by two spheres. This model leads to a further decrease of $\delta$ if the orientation of the L3 protein is chosen as is shown in Fig. 5 .

In a similar way the data of sample (3) lead to the position and orientation of L4 (Fig 5)

The radius of gyration for both L3 and L4 are about $19 \AA$ and they separated by a distance of $125 \pm 10 \AA$. A more detailed discussion will be given elsewhere ${ }^{[6]}$.

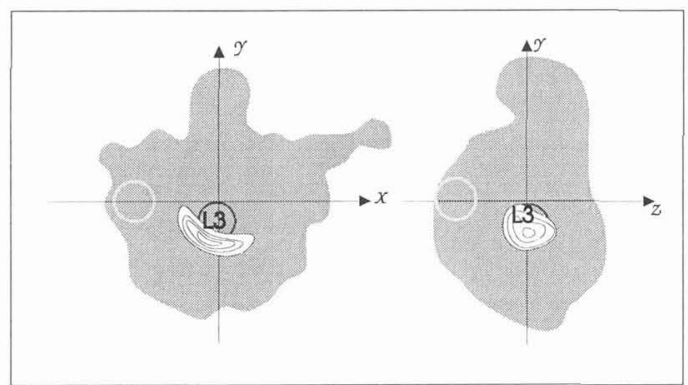

Fig. 4 The contours of equal deviations as given in eq.(2) for possible L3 locations around the minimum. Spherical model for L3 is assumed. The circles represent those determined by electromicroscopy ${ }^{[7]}$ and the shape of the $50 \mathrm{~S}$ is from Wagenknecht et al $^{[5]}$

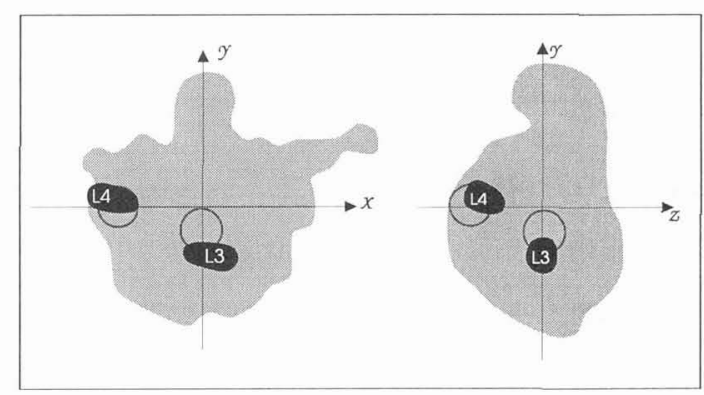

Fig. 5 The binding sites of the L3 and L4 proteins in the 50S subunit of $E$. Coli ribosome determined by Nuclear Spin Contrast Variation.

\section{Conclusion}

Nuclear spin contrast variation is a very powerful technique for the in situ structure determination of small components in large particles. From the present data it can be concluded that a fraction of 0.003 of the total particle still can be structurally characterized. The results presented here are not in contradiction to those obtained from isotopic substitution ${ }^{[8]}$ and microscopy ${ }^{[7]}$, However they provide structural information which could not be obtained by any other method before.

The authors are very much indebted to Tapio Niinikoski and Adriaan Rijllart at CERN, Geneva and Otto Schärpf at the ILL, Grenoble. The samples were prepared by Knud Nierhaus and his co-workers. at the MPI of Molecular Genetics, Berlin. The construction of the polarized neutron scattering facility with its polarized target station at GKSS, Geesthacht is supported by the BMFT, Bonn.

\section{References}

[1] Schärpf, O. Physica B 156\&157, 631 and 639 (1989).

[2] Nierhaus, K.H.: "Reconstitution of ribosomes" in Ribosomes and Protein Synthesis G. Spedding ed. 161-189 IRL Press Oxford (1990)

[3] Vanatalu, K. T. Paalme, R. Vilu, N. Burkhardt, R. Jünemann, R. May, M. Rühl, Jörg Wadzack, K. Nierhaus, Private commnication( to be published): "Large-Scale Prepaeration of Fully Deuterated Cell Components: Ribosomes from Escherichia coli with High Biological Activity".

[4] Abragam A., M. Goldman Nuclear Magnetism, Order and Disorder, Clarendon Press, Oxford(1982).

[5] Wagenknecht, T., Grassucci and J. Frank, J. Mol. Biol. 199 137-147 (1988).

[6] Zhao. J. N. Burkhardt, W. Meerwinck, K,'.H. Nierhaus, M. Rühl, M. Schmitt., H. Voss., R. Willumeit and H. Stuhrmann. (1993) To be published

[7] Stöffler-Meilicke M. and G. Stöffler The Ribosome, Structure, Function and Evolution, W. Hill ed.ASM, Washington DC, 123-133, (1990).

[8] May R.P., V. Novotny, P. Novotny, H. Voss, K.H. Nierhaus, EMBO Journal 11373 (1992). 